\title{
Conductance fluctuations due to a bistable scatterer in a weakly connected conductor
}

\author{
Vladimir I. Fal'ko \\ Max-Planck-Institut für Festkörperforschung, Heisenbergstrasse 1, 70569 Stuttgart, Germany \\ and Institute of Solid State Physics, Chernogolovka, 142432, Russia \\ (Received 28 July 1994; revised manuscript received 6 October 1994)
}

\begin{abstract}
We study the effect of a single bistable scatterer on the conductance of mesoscopic conductors in the crossover regime between open and closed systems. After the resistance of the contact between the metallic grain and bulk electrode exceeds the resistance of a grain, the effect of a bistability is enhanced and takes the universal form. Its efficiency scales with the cross section of the bistable impurity and conductances of contacts but is independent of this impurity position inside a sample. Mutual correlations between magnetoconductance fluctuations at two conductance levels also look universal. Enhancement of the conductance sensitivity to variations of impurity configurations in samples with highly resistive contacts transforms the spectral density of excess quantum noise toward a white-noise behavior and requires higher stability of the sample in order to observe mesoscopic effects in direct-current measurements.
\end{abstract}

\section{INTRODUCTION}

Mesoscopic devices of various modifications often show pronounced telegraph and other low-frequency noise. ${ }^{1}$ Some part of this noise has been interpreted ${ }^{2}$ in terms of the presence of bistable or multistable configurations of defects or of a slow impurity diffusion. The origin of this effect has a quantum nature. In the absence of any inelasticity and at low temperatures, the conductance of an individual sample depends on the interference pattern of diffusive electronic waves in it, ${ }^{3}$ and a bistable scatterer manifests itself by the change of phases of coherent multiply scattered electron waves which touch it during the pass through the sample. As a result, the recharging of even a single impurity among an infinite number of them can produce a finite effect on the conductance. ${ }^{2}$

Nevertheless, in devices with the form of a microbridge (open systems), this effect is rather limited. Recharging or displacement of a single short-range impurity among a lot of them (their density is $n$ ) produces a conductance variation of the order of $\left\langle\delta g^{2}\right\rangle \sim\left(l^{2} n\right)^{-1}$ (Ref. 2) (we use quantum units $e^{2} / h$ to measure all conductances below and brackets stand for an averaging over static impurity configurations). This cannot provide a complete renewing of a random potential realization from the point of view of a resulting transmission through the sample, since the number of impurities $n l^{2}$ per area of a squared mean free path $l$ is usually great. Further, both the absolute value and autocorrelation function of conductance variations in a magnetic field are quite sensitive to the bistable defect position with respect to current contacts. ${ }^{4}$ The goal of the present work is to describe the effect of a single scatterer on properties of a nearly closed mesoscopic conductor and, in particular, to estimate, how much changes one should produce in a disordered cavity with low-conductance leads in order to collect full statistics of conductance fluctuations (CF) in it. The latter information can be useful both for the studies of mesoscopic effects in electronic microdevices ${ }^{5,6}$ and transmission ex- periments with microwaves, ${ }^{7}$ which could be directed to the check of recently predicted universal distributions of transport coefficients of chaotic microcavities. ${ }^{8}$ The calculation below confirms an intuitive expectation that, as the mesoscopic conductor gets less and less connected to bulk electrodes (conductances $g_{b}$ of contacts decrease), the influence of an individual scatterer on the conductance increases. The crossover occurs when the contact resistance, $g_{b}^{-1}$, exceeds that of a metallic grain $\left(g_{c}^{-1}\right)$. After this, CF's produced by a bistability take the universal asymptotic behavior specific to zero-dimensional systems: their rms value, $\left\langle\delta g^{2}\right\rangle \sim g_{b}^{-1}\left(\sigma_{i} / \lambda_{F}^{d-1}\right)$ becomes independent both of the position of the bistable impurity and of the density of other scatterers, but depends on the impurity cross section $\sigma_{i}$ weighted by the electron Fermi wavelength $\lambda_{F}$ and the contact conductance. The normalized magnetocorrelation function between conductances related to different impurity states also takes the universal form resembling that of magnetofluctuations of the density of states in a closed chaotic billiard. ${ }^{10}$ The above statement is also partly valid at relatively high temperatures.

\section{CONDUCTANCE FLUCTUATIONS IN A WEAKLY CONNECTED DIFFUSIVE CONDUCTOR}

In order to study the mesoscopic conductance in the crossover regime between completely open ${ }^{3}$ and completely closed $^{9}$ chaotic systems, we consider the circuit composed of a diffusive coherent metallic conductor (with conductance $g_{c}$ ) connected to reservoirs by two equal, highly resistive contacts (with individual conductances $\left.g_{b}\right)$. The complication of calculations specific to the Coulomb blockade regime can be avoided by suggesting that the conductance $g_{b} \gg 1$. The same condition, reformulated in terms of energetic characteristics of levels, holds the inequality $\Gamma \gg \Delta \epsilon$ between the characteristic 
escape-associated width of states in a box, $\Gamma$, and their mean level spacing $\Delta \epsilon$. The latter relation is known to be a condition to the applicability of the perturbation theory analysis, ${ }^{9}$ so that in what follows, we slightly modify the conventional diagrammatic calculations ${ }^{3}$ into a form which allows us to incorporate the effect of resistive contacts. We do this in the fashion of earlier works. ${ }^{11,12}$

In a piece of a disordered metal $\left(L \gg l \gg \lambda_{F}\right)$, the electron density-density and phase-phase correlations obey the diffusion law, the same as the electron distribution function $N(\epsilon),\left(\partial_{t}-D \vec{\partial}^{2}\right) N(\epsilon)=0$, with the same diffusion coefficient $D$ and the same kind of boundary conditions. The latter are the continuity and current conservation equations which one should read as $\vec{n} D \vec{\partial} N=0$ at the wire boundaries and $( \pm 2 \alpha L) \partial_{x} N=$ $N-N_{F}(\epsilon \pm e V / 2)$ at the edges $x= \pm L / 2$, respectively. Here, $N_{F}$ is the Fermi distribution function, and we already exploited the quasi-one-dimensional wire geometry and separate the longitudinal $(x)$ and transverse variables. The meaning of the parameter $\alpha$ can be clarified after comparing the value of an averaged current through three resistors $\left(g_{b}^{-1}, g_{c}^{-1}\right.$ and again $\left.g_{b}^{-1}\right)$ in series with the value calculated from the diffusion equations using the definition of a total current as an integral of a local current density in a metallic part of this circuit, $\langle I\rangle=-e D L^{-1} \nu_{F} \int d \mathbf{x} d \epsilon\left[\partial_{x} N(\epsilon)\right]\left(\nu_{F}\right.$ is the density of states at the Fermi level and $L$ is the length of a wire). After comparison, we determine the parameter $\alpha$ as the ratio between the wire to contact conductances,

$$
\alpha=\frac{g_{c}}{2 g_{b}},
$$

so that an open system corresponds to $\alpha \rightarrow 0$ and a quasi-isolated one is described by the limit of $\alpha \gg 1$.

Using the above definition of the current through the circuit in terms of the integrated current density in its metallic part, the correlation function $K=\left\langle g_{1} g_{2}\right\rangle-\langle g\rangle^{2}$ of two conductances, $g_{1(2)}=g(U \pm \delta U / 2, H \pm \Delta H / 2)$, taken for different impurity configurations $U_{1,2}=U \pm$ $\delta U / 2$ and at different magnetic fields $H_{1,2}=H \pm \Delta H / 2$ can be related ${ }^{3}$ to two-particle Green functions $P_{d(c)}=$ $\left\langle G_{U_{1} H_{1}}^{R} G_{U_{2} H_{2}}^{A}\right\rangle$ averaged over realizations of a "background" random potential $U$, so called diffusons and Cooperons. The latter two describe the density-density and phase-phase correlations of electrons in a diffusive regime and, after accounting for the variation $\delta U$, they can be found from the equations ${ }^{4}$

$$
\begin{aligned}
\{-i \Omega & -D\left(\vec{\partial}-i \frac{e}{\hbar c} \vec{A}_{d(c)}\right)^{2} \\
& \left.+u \tau_{f}^{-1} V \delta\left(\vec{r}-\vec{r}_{i}\right)\right\} P_{d(c)}\left(\Omega, \vec{r}, \vec{r}^{\prime}\right)=\delta\left(\vec{r}-\vec{r}^{\prime}\right) .
\end{aligned}
$$

In this equation, the effect of a single bistable scatterer [placed at the coordinate $\vec{r}_{i}=\left(\xi_{i} L, \mathbf{r}_{\perp}\right)$ ] is incorporated into a $\delta$-functional repulsive potential with the strength

$$
u \approx\left(\frac{L}{l}\right)^{2} \frac{1}{V n} \frac{\sigma_{i}}{\sigma_{\mathrm{tr}}}
$$

where $V n$ is the total number of short-range scatterers inside the sample with volume $L \times S_{\perp}, \sigma_{\mathrm{tr}}$ - their transport cross section, $\sigma_{i}$ - the cross section of a varied impurity potential $\delta U$, and $\tau_{f}=L^{2} / D$. Equation (2) should be completed with the boundary conditions,

$$
\left\{\vec{n}\left(\vec{\partial}-i \frac{e}{\hbar c} \vec{A}_{d(c)}\right)\right\} P_{d(c)}=0
$$

at the surface, and

$$
P_{d(c)}=( \pm \alpha L)\left(\partial_{x}-i \frac{e}{\hbar c} A_{x, d(c)}\right) P_{d(c)}
$$

at contacts to reservoirs, $x= \pm L / 2$. Here, $\alpha$ has the same meaning as above, and one can see that the escapeinduced term in the boundary conditions on the twoparticle Green functions $P_{d(c)}$ is twice as efficient as that of a single-particle distribution function $N(\epsilon)$. For the sake of convenience, we choose such a gauge of the field $\vec{A}_{d(c)}, \operatorname{rot} \vec{A}_{d(c)}=\vec{H}_{1} \pm \vec{H}_{2}$, which provides us with $\vec{n} \vec{A}=0$ and allows an easy transition to quasi-one-dimensional formulas. In particular, the above equations can be applied to the lowest transverse diffusive mode and, therefore, we rewrite it in the form where the presence of a gauge field in a long derivative, $\left(\vec{\partial}-i \frac{e}{\hbar c} \vec{A}\right)^{2}$, is replaced by an extra decay rate $\gamma^{2}$ : we get $\left(\partial_{x}^{2}-\gamma^{2} / L^{2}\right)$, where $\gamma_{d(c)}^{2}=\frac{(2 \pi)^{2}}{3}\left(\phi_{1} \pm \phi_{2}\right)^{2}$ is determined by the value of a magnetic field fluxes, $\phi_{1,2}=S H_{1,2} / \Phi_{0}$, through sample area, and $\Phi_{0}=h c / e$.

If we restrict the analysis to the case $g_{b} \gg 1$, we can confine the perturbation theory calculation of $K=$ $\left\langle g_{1} g_{2}\right\rangle-\langle g\rangle^{2}$ to the diagrams ${ }^{3}$ which contain two diffusons or Cooperons, so that

$$
\begin{aligned}
K= & \frac{4 D^{2}}{L^{2}} \int d \epsilon d \epsilon^{\prime} \int d x d x^{\prime}\left[\partial_{x} \partial_{\epsilon} N(\epsilon)\right]\left[\partial_{x} \partial_{\epsilon^{\prime}} N\left(\epsilon^{\prime}\right)\right] \\
& \times \sum_{d(c)}\left\{\left|P_{d(c)}\left(\Omega ; x, x^{\prime}\right)\right|^{2}+\frac{1}{2} \operatorname{Re} P_{d(c)}^{2}\left(\Omega ; x, x^{\prime}\right)\right\},
\end{aligned}
$$

where $\Omega=\epsilon-\epsilon^{\prime}$. (The diagrams of higher orders in $P_{d(c)}$ are suppressed by the factor $g_{b}^{-1}$.) After substituting $N$ and $P_{d(c)}$ from Eq. (2) into Eq. (3), the latter can be reduced to the form $K\left(u, \phi_{1}, \phi_{2}\right)=K_{d}+K_{c}=$ $K(u, \Delta \phi)+K(u, 2 \phi)$,

$$
K(u, \tilde{\phi})=\frac{6}{(1+4 \alpha)^{2}} \sum_{q_{n}} \frac{1}{\left[q_{n}^{2}+\gamma^{2}(\tilde{\phi})\right]^{2}} .
$$

In deriving Eq. (4) and in finding the set of $q_{n}$ 's which stay in this expression, we treat $P_{d(c)}$ as the Green functions of Eqs. (2), which are similar to the Schrödinger equation of a particle in a corresponding potential,

$$
P\left(\Omega ; x, x^{\prime}\right)=\sum_{n} \psi_{n}(x) \psi_{n}\left(x^{\prime}\right) /\left(-i \Omega+\Omega_{n}\right)
$$

Here, $\psi_{n}$ are the eigenfunctions and $\Omega_{n}=D q_{n}^{2}$ are the eigenvalues of the spectral problem given by Eq. (2) with its boundary conditions. For the case of a single bistable scatterer in a quasi-one-dimensional wire placed at $x_{i}=$ $\xi_{i} L$ we write $\psi_{n}^{l(r)}=a_{l(r)} \cos \left(q_{n} x+\theta_{l(r)}\right)+b_{l(r)} \sin \left(q_{n} x+\right.$ $\left.\theta_{l(r)}^{\prime}\right)$, where the indices $l(r)$ denote the wire intervals to the left (right) from the scatterer $\xi_{i} L$. Using the analogy between the generalized diffusion equation (2) and the Schrödinger equation, we repeat the conventional way ${ }^{13}$ to find the spectrum of $q$ 's. That is, the coefficient $a$ and $b$, the phases $\theta^{\prime}$ s and the spectrum of $q$ 's have to be 
found from the above-mentioned boundary condition at the wire edges and after adjusting functions $\psi^{l}$ and $\psi^{r}$ at the scatterer position: $\psi$ has to be continuous in this point and the jump of its derivative $\partial_{x} \psi$ is determined by the strength of the $\delta$ potential in Eq. (2). This gives one the set of linear equations. After eliminating the coefficients $a, b, \theta$, and $\theta^{\prime}$ from the set of linear equations, we arrive at the eigenvalue equation $F(q)=0$ on the number of wave modes $q_{n}$.

Skipping the details of this linear algebraic procedure, we find that for samples with the form of a single wire,

$$
F(q)=2 \cos q+\frac{\sin q}{\alpha q}-\alpha q \sin q+u \alpha f .
$$

In this expression the function $f$ includes all the necessary parameters of a bistable scatterer,

$$
\begin{aligned}
f= & \frac{\sin q}{\alpha q}+\frac{\cos q+\cos \left[\left(1-2 \xi_{i}\right) q\right]}{2} \\
& -\frac{\cos \left[\left(1-2 \xi_{i}\right) q\right]-\cos q}{2(\alpha q)^{2}} .
\end{aligned}
$$

In the case of a ring (Aharonov-Bohm) geometry, ${ }^{14}$ the function $F$ can be derived from the same set of equation as before, but with the boundary conditions on the electron diffusion in two semicirclets (with a total conductance $g_{c}$ ) connected near contacts. It has the form

$$
\begin{aligned}
F(q)= & \frac{1-\cos (2 q)}{(\alpha q)^{2}}+\cos (2 q) \\
& -\cos (2 \pi \tilde{\phi})+\frac{2 \sin (2 q)}{\alpha q}+\frac{\alpha u}{2} f
\end{aligned}
$$

where

$$
\begin{aligned}
f= & \frac{\sin (2 q)}{\alpha q}+\sum_{ \pm}\left[\frac{\cos \left[\left(1 \pm 2 \xi_{i}\right) q\right]-\cos (2 q)}{(\alpha q)^{2}}\right. \\
& \left.+\frac{\sin \left[\left(1 \pm 2 \xi_{i}\right) q\right]-\sin (2 q)}{(\alpha q)^{3}}\right]
\end{aligned}
$$

and $\tilde{\phi}$ denotes the magnetic field flux encircled by a ring and measured in units of $\Phi_{0}$.

The summation over the set of eigenvalues $q_{n}$ can be performed using the following procedure. We rewrite the sum in Eq. (4) as a result of an integration of the function $R(z)=F^{\prime}(z) /\left[F(z)\left(z^{2}+\gamma^{2}\right)^{2}\right]$ over a complex variable $z$ along the contour $C$ "encircling" the real axis. Function $R(z)$ has poles $z= \pm q_{n}$ on the real axis, two poles $z=$ $\pm i \gamma$ on the imaginary axis and tends to zero at $z \rightarrow \infty$. Therefore, it is natural to shift the contour $C$ to $\pm i \infty$, so that the value of the correlation function $K$ would be determined by the residues of $R(z)$ at $z= \pm i \gamma$. This gives us

$$
K(u, \phi)=\frac{6}{(1+4 \alpha)^{2}} \operatorname{Re}\left\{\frac{d}{d z}\left(\frac{-F^{\prime}(z)}{F(z)(z+i \gamma)^{2}}\right)_{z=i \gamma}\right\}
$$

\section{UNIVERSAL CORRELATION FUNCTION OF CONDUCTANCE FLUCTUATIONS IN NEARLY CLOSED SYSTEMS}

Now one can describe the correlations $K\left(u, \phi_{1}, \phi_{2}\right)$ at any value of parameters involved and analyze the asymp- totic limits. In particular, we can follow the features of magnetoconductance fluctuations from open $(\alpha \rightarrow 0)$ to closed $(\alpha \rightarrow \infty)$ systems. In an open system, we arrive at the well known rms value of the universal $\mathrm{CF}$ (Refs. 3 and 8) and the correlation function, $K\left(\phi_{1}, \phi_{2}\right)=$ $K(2 \phi)+K(\Delta \phi)$, with

$$
K= \begin{cases}\frac{3}{2} \frac{2 \gamma^{2}+2+\gamma \sinh (2 \gamma)-\cosh (2 \gamma)}{\gamma^{4}[\cosh (2 \gamma)-1]}, & \text { wire } \\ \frac{1}{15} \frac{1+2 \alpha^{2} \cos (2 \pi \tilde{\phi})}{\left[1-\frac{1}{2} \alpha^{2} \cos (2 \pi \tilde{\phi})\right]^{2}}, & \text { ring }\end{cases}
$$

which describes both wire and ring geometries.

In an opposite limit of a weak connection $(\alpha \gg 1)$, we get a different geometrical prefactor in the rms value of CF in the unitary limit, $\operatorname{var}(g)=\frac{3}{32}$, and find the flux-dependent correlation function of a ring $K\left(\phi_{1}, \phi_{2}\right)=$ $K(2 \phi)+K(\Delta \phi)$

$$
K(\tilde{\phi})=\frac{1}{16} \frac{1+\frac{1}{2} \cos (2 \pi \tilde{\phi})}{\left(1+\frac{\alpha}{4}[1-\cos (2 \pi \tilde{\phi})]\right)^{2}},
$$

which shows periodically repeated narrow splashes in the form of squared Lorentzians of a width $\Phi_{0} / \sqrt{\alpha}$.

The effect of a single bistable impurity on a sample conductance can be analyzed from the dependence of the correlation function $K$ on the parameter $u$. After studying the limit of $\alpha \rightarrow 0$ (open system), one can see that the effect of a single scatterer on a conductance can never be strong enough to renew completely the realization of a random potential configuration. Second, the jump in the conductance value due to a change of one impurity shows a dependence (through the parameter $\rho=\frac{1}{4}-\xi_{i}^{2}$ ) on its position relative to the wire edges, both in the bistable-scatterer efficiency,

$$
1-\frac{K(u)}{\operatorname{var}(g)}=2 \rho^{2} u \frac{2(1+\rho u)+\rho(4-\rho u)}{(1+\rho u)^{2}},
$$

and in autocorrelation properties under a variation of a magnetic field. The latter could even allow one to make a rough tomography of a bistable impurity. ${ }^{4}$

In a system with highly resistive contacts, $\alpha \gg 1$, the electron spends much longer time inside the mesoscopic conductor, as compared to the diffusion time $\tau_{f}=L^{2} / D$. A classical diffusive trajectory in a "box" is much longer than in an open system, so that it has a better possibility of meeting a bistable scatterer many times during different traversals from one contact to another. This produces its higher efficiency in renewing the sample realization and results in the universal form of the correlation function $K\left(u, \phi_{1}, \phi_{2}\right)$,

$$
\begin{aligned}
\frac{K\left(u, \phi_{1}, \phi_{2}\right)}{\operatorname{var}(g)}= & \left(1+\frac{\alpha}{2}\left[u+\gamma_{d}^{2}(\Delta \phi)\right]\right)^{-2} \\
& +\left(1+\frac{\alpha}{2}\left[u+\gamma_{c}^{2}(2 \phi)\right]\right)^{-2}
\end{aligned}
$$

The first term in the right hand side describes correlations between $g_{1}$ and $g_{2}$ in the unitary limit. The second can be used for discussing the crossover regime to the orthogonal $(H=0)$ ensemble. The parameter $u=\left(\frac{L}{l}\right)^{2} \frac{1}{V_{n}} \frac{\sigma_{i}}{\sigma_{\mathrm{tr}}}$ related to a change of a scatterer enters into this expression in combination with enhancement parameter $\alpha=g_{c} / 2 g_{b}$. After using the Drude-Einstein formula, we find that the effect of a single scatterer is 
scaled by the combination of its scattering cross section and the conductance $g_{b}$,

$$
\alpha u \approx \frac{1}{g_{b}} \frac{\sigma_{i}}{\lambda_{F}^{d-1}}
$$

Remarkably, this combination does not depend on properties of other scatterers, except that we think about the limit of $\lambda_{F}<l<L$.

Equations $(7,8)$ tell us how many scatterers have to be changed-shifted in order to collect the full statistics of transmission fluctuations through a quasiclosed quantum box. In electronic devices prepared of metals with short-range scatterers, the effect of single impurity is never strong enough until the system becomes completely closed and gets into the Coulomb blockade regime. In low-density semiconductor devices, the displacement of a trapped charge near the two-dimensional (2D) channel affects electron scattering phases more than a short-range defect can do it. A rough estimation shows that, after $2 \mathrm{D}$ screening at the distance of a donor-related Bohr $a_{B}$ is taken into account, a single recharging event can be sufficient enough if $g_{b} \sim \lambda_{F} / a_{B}$. As applied to microwave experiments on a single mode transmission through diffusive cavities (that corresponds to $g_{b}=1$ ), Eqs. $(7,8)$ show that a single scatterer (such as a small piece of a metal) can already produce enough changes, if its size is of about a wavelength of irradiation.

The multiple diffusive traversal of a quasiclosed system by an electron-from contact to contact-destroys the memory about a specific impurity position, which makes the magneto-tomography of a bistable scatterer no longer possible. This is manifested by the fact that the correlation function of random magnetoconductance variations takes the universal form Eq. (6), which is similar to that of thermodynamic parameters of isolated systems, ${ }^{10}$ but with a rescaled correlation magnetic field flux $\phi_{c}=H_{c} S$. The field $H_{c}$ in a weakly connected mesoscopic conductor lies in between of what is known for open systems, $\phi_{c}(\alpha \rightarrow 0)=\Phi_{0}$, and $\phi_{c}(\Gamma<\delta \epsilon)=\Phi_{0} \sqrt{\delta \epsilon / E_{c}}$ determined by the mean level spacing $\delta \epsilon$ in isolated metallic grains. ${ }^{9,10}$ Since the lifetime of a diffusive electron inside a weakly connected conductor (the quantity inverse to $\Gamma / h)$ is $\alpha \gg 1$ times longer than the diffusive flight time $\tau_{f}=L^{2} / D$, the mean square $\left\langle\phi^{2}\right\rangle$ of a magnetic field flux encircled by a characteristic diffusive chaotic trajectory is $\alpha$ times greater than the geometrical flux $S H$ through the sample area. This rescales ${ }^{12,15,16}$ the correlation magnetic field flux of CF, in our case-down to the value of $\phi_{c}=\Phi_{0} /(\pi \sqrt{\alpha}) \ll \Phi_{0}$.

In a weakly connected conductor, a finite temperature shows up at the scale of $2 \pi T \geq \Gamma=h \tau_{f}^{-1} / \alpha \sim E_{c} / \alpha$. Thermal smearing of the Fermi distribution function beyond a shortened correlation energy $\Gamma=\alpha^{-1} h \tau_{f}^{-1}$ partially cancels CF coming from "independent" spectral intervals. At those high temperatures, the correlation function $K$ can be calculated from Eq. (3) by transforming the integral over energies into the sum over Matsubara's frequencies, and at $\alpha \gg 1$, we arrive at

$$
\frac{K(u, \Delta \phi)}{\operatorname{var}(g)}=\frac{\Gamma}{9 T}\left(1+\frac{\alpha}{2}\left[u+\gamma_{d}^{2}(\Delta \phi)\right]\right)^{-1}
$$

which again has universal parametric dependence. ${ }^{17}$

Since in earlier experiments ${ }^{5,6,18}$ the magnetocorrelations of conductance fluctuations were also studied using the Fourier transform representation of the data, it is reasonable to compare the mean square values of the Fourier components of conductance fluctuations in a magnetic field with what one can get from Eqs. (6) and (8). rms values of a Fourier transform of $A B$ oscillations in a ring with respect to the flux $\phi$ shows directly the efficiency of the electron escape from a sample: $\left\langle\delta g_{k}^{2}\right\rangle \propto(\alpha / 2)^{2 k}$, $\alpha \ll 1$. In a weakly connected sample, $\alpha \gg 1$,

$$
\left\langle\delta g_{k}^{2}\right\rangle=\frac{\operatorname{var}(g)}{\sqrt{2 \alpha}}\left(1-\frac{2 \sqrt{2}}{\sqrt{\alpha}}\right)^{k} \kappa(T / \Gamma),
$$

where the most serious temperature dependence of the "spectral function" is incorporated into the multiplyer $\kappa$. In two limits of a low and high temperatures, $\kappa(0)=1$ and $\kappa(T \gg \Gamma)=\frac{2 h \tau_{f}^{-1}}{9 T \alpha} \cdot{ }^{17}$ This behavior is consistent with the rms value of the Fourier transform of $\mathrm{CF}$ with respect to the flux $2 \pi \phi$ through the sample of a singleconnected geometry: At $\alpha \gg 1$, we get

$$
\left\langle|\delta g(k)|^{2}\right\rangle=\operatorname{var}(g) \frac{\pi}{b} \frac{\kappa(T / \Gamma)}{\sqrt{2 \alpha}} e^{-b k \sqrt{2} / \sqrt{\alpha}},
$$

where $b \sim 1$ is a geometrical factor. The factor $\kappa(T / \Gamma)$ is the same as above. One can see that the exponential dependence of the Fourier transform on the "frequency" $k$ of random magnetoconductance oscillations is similar for the low and high temperatures, like it has been observed in (Refs. 5 and 6).

\section{BISTABLE-SCATTERER-INDUCED EXCESS NOISE IN A QUANTUM CONDUCTOR}

Higher sensitivity of nearly closed systems to a change of the impurity potential causes modifications of the spectral shape of the low-frequency excess noise (generated by random switches of several, $\delta N_{i} \gg 1$, localized charges or defects) towards the white-noise dispersion. That is, a variation of scatterers at the characteristic time scale $\tau_{r},\langle U(0) U(t)\rangle \propto \kappa\left(t / \tau_{r}\right)$, related to a spectral dispersion of the source of the noise at the frequency $\omega_{r} \sim \tau_{r}^{-1}$ destroys the correlation of instant conductance values, $K(t) \sim\left\{1+\frac{\alpha u}{2}\left[1-\kappa\left(t / \tau_{r}\right)\right]\right\}^{-2}$, at a much shorter time scale: $t_{*}=\tau_{r} \tau_{i} /\left(\alpha \tau_{f}\right)$. This estimation can be produced in the same way as in Ref. 19. In order to do this, we introduce the scattering rate $\tau_{i}^{-1}=u \tau_{f}^{-1} \propto \delta N_{i}$ instead of the parameter $u$. The rate $\tau_{i}^{-1}$ shows us how often the diffusive electron scatters on a bistable impurity, so that the ratio $\alpha \tau_{f} / \tau_{i} \gg 1$ gives the probability that an electron meets this scatterer during this lifetime inside a sample. Therefore, the time $t_{*}$ can be obtained from the condition $\left(t_{*} / \tau_{r}\right)\left(\alpha \tau_{f} / \tau_{i}\right) \sim 1$, which requires that each diffusive path can meet a renewed scatterer with a unit probability, and this will be the value which determines the spectral shape of a low-frequency excess noise in a current. By the definition, the latter is 
$S(\omega)=\int d t e^{i \omega t}\langle I(t) I(0)\rangle$ and can be found as

$$
S(\omega) \sim\left(\frac{e^{2} V}{h}\right)^{2}{ }_{\frac{\tau_{r} \tau_{i}}{\alpha \tau_{f}}}\left\{\begin{array}{c}
1, \omega<1 / t_{*} \\
\left(\omega t_{*}\right)^{-2}, \omega>1 / t_{*} .
\end{array}\right.
$$

From this, as more breathing impurities are located inside a metallic region, the spectral density of a noise is redistributed over a range of frequencies $\sim 1 / t_{*}$ much wider than spectral range of the source of a noise (the noise is transformed to the white noise). It is also clear that the integral intensity $\int d \omega S(\omega)=\delta I(t)^{2} \sim\left(e^{2} V / h\right)^{2}$ remains fixed.

On the other hand, the above consideration shows that the observation of effects of coherence in direct-current measurements in a closed system requires a higher stability of samples, as compared to open ones. Any internal source of a soft noise reduces the amplitude of mesoscopic CF down to the value $\operatorname{var}(g)=K(t \rightarrow \infty)$. This would be, for example, crucial for their observation in (even slightly) magnetically contaminated systems. That is, the electron spin-flip scattering makes conductance dependent on an instant configuration of spins of few magnetic impurities, whereas the Korringa relaxation of impurity spins - caused by the same flip-flop with thermal or current electrons- varies the transmission in time. ${ }^{19,20}$ This effect is completely analogous to the impurity recharging or displacement discussed above and leads to the same result: The mesoscopic dc conductance fluctuations are easily washed out even when the spinflip scattering length in a bulk material, $l_{i}=\left(D \tau_{i}\right)^{1 / 2}$, is much longer than sample dimensions. Therefore, at low magnetic fields, dc fluctuations are suppressed down to the rms value

$$
\operatorname{var}(g) \sim\left(\frac{\tau_{i}}{\alpha \tau_{f}}\right)^{2}=\left(\frac{l_{i}}{L \sqrt{\alpha}}\right)^{4} \ll 1 .
$$

A spectacular feature of a paramagnetic impurity system can be expected after application of a high enough magnetic field. If the latter is able to polarize spins of scatterers, their time-dependent variation would be suppressed which can be observed as an abrupt restoration of the universal CF's in dc measurements.

\section{CONCLUSIONS}

In a summary, we have analyzed the conductance fluctuations in a mesoscopic piece of a metal in the crossover regime from an open to a nearly closed system. On the base of the perturbation theory calculations, we conclude that after the resistances $g_{b}^{-1}$ of the device contacts to bulk electrodes exceed the resistance $g_{c}^{-1}$ of a metallic grain itself, the sensitivity of a sample conductance to the variation of a bistable scatterer increases, so that at $g_{b} \sim 1$ even a single scatter can produce a variation $\delta g \sim 1$. In the same limit, the correlations function of conductance fluctuations takes the universal form specific to the zero-dimensional system, both with respect to the variation of an impurity configuration and external parameters, such as a magnetic field.

Although the calculations are performed in the diffusion regime, the derived above magnetocorrelation functions seem to be able to describe fluctuations in chaotic ballistic systems. In particular, Eqs. $(7,9,10)$ are in a good agreement with observations of Refs. 5, 6 and 18. One can also expect that in billiards the variation of an impurity can be replaced by the variation of a shape, but before using the derived above equations one has to take into account the following remark. The variation of a shape is usually produced by some variation of a voltage $\delta V_{g}$ applied to side gates. ${ }^{5}$ This variation of gate voltages changes not only the shape of a structure, but also its area, $S \rightarrow S+\delta S$. In an arbitrary case, the change of an area occurs in the first order on $\delta V_{g}$, $\delta S \propto \delta V_{g}$, and results in a proportional rescaling of the energies $\epsilon_{m}$ of all single-particle states in the box spectrum: $\epsilon_{m} \rightarrow \epsilon_{m} /(1+\delta S)$. Therefore, the states near the Fermi level flow together through the Fermi level roughly preserving their spatial structure, and, therefore, their transport abilities, since the change of a scattering due to a direct shape variation can appear only as a quadratic term on $\delta V_{g}$. This results in the same effect as if one would vary the Fermi energy itself ${ }^{18}$ and has to be described by the correlation function,

$$
\frac{K}{\operatorname{var}(g)}=\left(\left[1+\frac{\alpha}{2} \gamma^{2}(\Delta H)\right]^{2}+\left[\frac{\alpha \tau_{f} \delta \epsilon}{h}\right]^{2}\right)^{-1},
$$

where $\delta \epsilon \propto \delta S \propto \delta V_{g}$. It is amusing to mention that, after necessary substitutions, the parameter $\alpha \tau_{f} \delta \epsilon / h$ which stands in the function $K$ can be rewritten as $\delta N_{e} / g_{b}$, where $\delta N_{e}$ is an actual change of a number of carriers assigned to the interior of a metallic box.

\section{ACKNOWLEDGMENTS}

This work has been stimulated by discussions with J.C. Maan of experiments on the transmission through disordered microwave cavities. I also thank K.B. Efetov for comments on universality in zero-dimensional systems and information concerning his work (Ref. 16) and C. Marcus for discussions of the shape-variation effects in ballistic quantum billiards (Ref. 6). Partial support from ISF Grant REE-000 and NATO Collaborative Research Grant No. 921333 is acknowledged.
${ }^{1}$ M.J. Kirton and M.J. Uren, Adv. Phys. 38, 367 (1989); M.B. Weismann, Rev. Mod. Phys. 60, 537 (1988); 65, 829 (1993), and references therein.

${ }^{2}$ B.L. Altshuler and B.Z. Spivak, Pis'ma Zh. Eksp. Teor. Fiz. 42, 363 (1985) [JETP Lett. 42, 447 (1985)]; S. Feng et al., Phys. Rev. Lett. 56, 1960 (1986).

${ }^{3}$ B.L. Altshuler, Pis'ma Zh. Eksp. Teor. Fiz. 41, 530 (1985) [JETP Lett. 41, 648 (1985)]; P.A. Lee and A.D. Stone, Phys. Rev. Lett. 55, 1622 (1985); A.D. Stone, ibid. 54, 2692 (1985); B.L. Altshuler and D.E. Khmel'nitskii, Pis'ma 
Zh. Eksp. Teor. Fiz. 42, 291 (1985) [JETP Lett. 42, 359 (1985)]; D.E. Khmel'nitskii and A.I. Larkin, Phys. Scr. T14, 4 (1986).

${ }^{4}$ V.I. Fal'ko and D.E. Khmel'nitskii, Pis'ma Zh. Eksp. Teor. Fiz. 51, 166 (1990) [JETP Lett. 51, 189 (1990)]; G. Bergmann, Phys. Rev. B 49, 8377 (1994).

${ }^{5}$ C.M. Marcus et al., Phys. Rev. Lett. 69, 506 (1992); Phys. Rev. B 48, 2460 (1993).

${ }^{6}$ I.H. Chan, R.M. Clarke, C.M. Marcus, K. Campman, and A.G. Gossard (unpublished); R.M. Clarke, I.H. Chan, C.I. Duruoz, J.S. Harris, C.M. Marcus, K. Campman, and A.G. Gossard (unpublished).

${ }^{7}$ H.J. Stockmann and J. Stein, Phys. Rev. Lett. 64, 2215 (1990); E. Doron et al., ibid. 65, 3072 (1990); H.-D. Graf et al., ibid. 69, 1296 (1992); A. Kudrolli et al., Phys. Rev. E 49, 11 (1994).

${ }^{8}$ V.N. Prigodin et al., Phys. Rev. Lett. 71, 1230 (1993); C.W.J. Beenakker and B. Rejaei, ibid. 71, 3689 (1993); J.T. Chalker and A.M.S. Macedo, ibid. 3693 (1993); H.U. Baranger and P.A. Mello, ibid. 73, 142 (1994).

${ }^{9}$ K.B. Efetov, Adv. Phys. 32, 53 (1983).
${ }^{10}$ A. Szafer and B.L. Altshuler, Phys. Rev. Lett. 70, 587 (1993); B.D. Simons and B.L Altshuler, ibid. 70, 4063 (1993); Phys. Rev. B 48, 5422 (1993).

${ }^{11}$ B.Z. Spivak and D.E. Khmel'nitskii, Pis'ma Zh. Eksp. Teor. Fiz. 35, 334 (1982) [JETP Lett. 35, 412 (1982)]; P. Santhanam, Phys. Rev. B 39, 2541 (1989).

12 R.A. Serota et al., Phys. Rev. B 35, 5031 (1987); V.I. Fal'ko, J. Phys. Condens. Matter 4, 3943 (1992).

${ }^{13}$ L.D. Landau and E.M. Lifshitz, Quantum Mechanics (Pergamon Press, Oxford 1965), p. 65.

${ }^{14}$ Y. Gefen et al., Phys. Rev. Lett. 52, 129 (1984); M. Buttiker et al., Phys. Rev. A 30, 1982 (1984)

${ }^{15}$ R.A. Jalabert et al., Phys. Rev. Lett. 68, 3468 (1992).

${ }^{16}$ K.B. Efetov, Phys. Rev. Lett. (unpublished).

17 At high temperatures, $K_{\text {ring }}(\phi)=\frac{\pi \Gamma}{96 T}\left(1+\frac{\alpha}{4}[1-\cos (2 \pi \phi)]\right)^{-1}$.

${ }^{18}$ M.W. Keller et al., Surf. Sci. 305, 501 (1994).

${ }^{19}$ A.A. Bobkov, V.I. Fal'ko, and D.E. Khmel'nitskii, Zh. Eksp. Teor. Fiz. 98, 703 (1990) [JETP 71, 393 (1990)].

${ }^{20}$ V. Chandrasekhar et al., Phys. Rev. B 42, 6823 (1990). 